\title{
Gauss's Law, Duality, and the Hamiltonian Framework of Lattice Gauge Theory
}

\author{
Jesse R. Stryker* \\ Institute for Nuclear Theory \\ University of Washington \\ Seattle, WA, USA \\ E-mail: strykereuw.edu \\ David B. Kaplan \\ Institute for Nuclear Theory \\ University of Washington \\ Seattle, WA, USA \\ E-mail: dbkaplan@uw.edu
}

\begin{abstract}
Quantum computers have the potential to solve certain problems in lattice gauge theory that are thought to be exponentially hard for classical computers. The proposed starting point for such computations has been the Kogut-Susskind Hamiltonian supplemented by the Gauss law constraint, with a cutoff on electric field values. There are several disadvantages to this approach, including having to simulate the vast unphysical part of the Hilbert space. We consider pure U(1) gauge theory, and, motivated to restrict the calculation to purely physical states, are immediately led to a duality transformation. We highlight the potential advantages this formulation of lattice gauge theory could have for simulations on quantum computers.
\end{abstract}

The 36th Annual International Symposium on Lattice Field Theory - LATTICE2018

22-28 July, 2018

Michigan State University, East Lansing, Michigan, USA.

${ }^{*}$ Speaker. 
Wilson's path integral construction provides a nonperturbative definition of lattice gauge theory and an efficient computational tool for some types of calculations. However, even for Quantum Chromodynamics (QCD) many properties lack a first-principles understanding, such as the phase diagram at finite chemical potential, real-time dynamics, topological properties, and the structure of all but the lightest nuclei. Such investigations entail sign problems that are exponentially hard to solve on a classical computer. Quantum computers offer hope for surmounting these obstacles and a number of papers have proposed using the Kogut-Susskind [1] lattice Hamiltonian $H_{\mathrm{KS}}$ as a starting point for the study of gauge theories, introducing a cutoff on the electric field in order to render the Hilbert space $\mathcal{H}$ finite-dimensional [2, 3, 4]. The vast majority of states in $\mathcal{H}$ are unphysical; the physical space is limited to those obeying Gauss's Law, [5] which we call $\mathcal{H}_{\text {phys }} \subset \mathcal{H}$.

There are a couple of drawbacks to this approach which we address here: (i) It appears preferable to work entirely in $\mathcal{H}_{\text {phys }}$ if possible, in order to require fewer qubits and to avoid computational errors causing states initially in $\mathcal{H}_{\text {phys }}$ to evolve into the large space of unphysical states; (ii) A cutoff on electric fields is appropriate for strong coupling, for which electric fluctuations are suppressed, but is not ideal for weak coupling, such as one would encounter in the continuum limit for any gauge theory in $d<3$, or asymptotically-free theories in $d=3$, where $d$ is the spatial dimension. Instead, a cutoff on magnetic fluctuations would likely be a more efficient regulator, allowing one to approach the continuum limit with a smaller Hilbert space. In this work we examine these issues in two of the simplest gauge theories - $\mathrm{U}(1)$ theories without matter in $d=2$ and $d=3-$ and find that both concerns lead directly to a formulation of the electromagnetic dual theory. While these theories are not of direct physical interest, they are simple enough to clearly illustrate some of the issues that must be faced when simulating $\mathrm{U}(1)$ gauge theories with matter, or non-Abelian gauge theories.

\section{U(1) Hamiltonian and Hilbert space}

The continuum Hamiltonian for a $\mathrm{U}(1)$ gauge theory is $\hat{H}=(1 / 2) \int d^{d} x\left(\hat{E}^{2}+\hat{B}^{2}\right)$, where the electric field $\hat{E}_{i}$ is the momentum conjugate to the vector potential $\hat{A}_{i}$. Here we consider compact $\mathrm{U}(1)$ gauge theory formulated on a spatial lattice $L$ with lattice spacing $a_{s}$, periodic boundary conditions, and coordinates $\{\mathbf{n}, \ell, \boldsymbol{p}, \boldsymbol{c}\}$ for sites, links, plaquettes, and cubes, respectively. Compact $\mathrm{U}(1)$ theory is interacting at finite $a_{s}$; because time and space are treated asymmetrically, there are two coupling constants $g_{t, s}$ which must be independently renormalized, with dimensionless couplings defined as $\tilde{g}_{t, s}^{2}=a_{s}^{3-d} g_{t, s}^{2}$. The continuum limit is equivalent to $\tilde{g}_{t, s}^{2} \rightarrow 0$ for $d<3$ (as well as for asymptotically-free non-Abelian gauge theories in $d=3$ ). We fix $A_{0}=0$ gauge, and replace the vector potential $\mathbf{A}(x)$ by a unitary operator $\hat{U}_{\ell}=\exp \left(i g_{s} a_{s} \hat{A}_{\ell}\right)$ on every link. The space $\mathcal{H}$ can be represented in the coordinate basis of product states $\otimes_{\boldsymbol{\ell}}\left|U_{\boldsymbol{\ell}}\right\rangle$, where $\left|U_{\boldsymbol{\ell}}\right\rangle$ at each link $\boldsymbol{\ell}$ diagonalize $\hat{U}_{\ell}$. Alternatively, one can work in a momentum basis that diagonalizes the electric field $\hat{E}_{\ell}$ also residing on the links. The two operators are related by the commutation relation

$$
\left[\hat{\mathscr{E}}_{\ell}, \hat{U}_{\ell^{\prime}}\right]=\delta_{\ell, \ell^{\prime}} \hat{U}_{\ell}, \quad \hat{\mathscr{E}}_{\ell} \equiv a_{s}^{\frac{d+1}{2}} \hat{E}_{\ell} / \tilde{g}_{s}
$$

where the rescaled field $\hat{\mathscr{E}}_{\ell}$ has integer eigenvalues $\varepsilon_{\ell}$. $\mathcal{H}$ can then be described using the electric field basis of product states $\otimes_{\ell}\left|\varepsilon_{\ell}\right\rangle$ and regulated in a gauge-invariant way by restricting fluctuations of the electric field, $\left|\varepsilon_{\ell}\right| \leq N$ for some cutoff $N$ [2]. 
Our starting point for the lattice Hamiltonian is $\hat{H}=\hat{H}_{E}+\hat{H}_{B}$, with

$$
\hat{H}_{B}=\frac{1}{2 a_{s}} \frac{1}{\tilde{g}_{s}^{2}} \sum_{\boldsymbol{p}}\left(2-\hat{P}_{\boldsymbol{p}}-\hat{P}_{\boldsymbol{p}}^{\dagger}\right), \quad \hat{H}_{E}=\frac{1}{2 a_{s}} \frac{\tilde{g}_{t}^{2}}{\xi^{2}} \sum_{\ell}\left(2-\hat{Q}_{\ell}-\hat{Q}_{\ell}^{\dagger}\right),
$$

and where we define

$$
\hat{Q}_{\ell} \equiv e^{i \xi \hat{\mathscr{E}}_{\ell}}, \quad \hat{P}_{\mathbf{n}, i j} \equiv \hat{U}_{\mathbf{n}, i} \hat{U}_{\mathbf{n}+\mathbf{e}_{i}, j} \hat{U}_{\mathbf{n}+\mathbf{e}_{j}, i}^{\dagger} \hat{U}_{\mathbf{n}, j}^{\dagger} .
$$

Here $\hat{H}_{B}$ is conventional with $\hat{P}_{\boldsymbol{p}}$ being the usual plaquette operator, but in $\hat{H}_{E}$ we have introduced the dimensionless parameter $\xi$ for convenience; eq. (1.2) yields $\hat{H}_{\mathrm{KS}}$ in the limit $\xi \rightarrow 0$. This Hamiltonian is similar to that of $Z(N)$ gauge theory in [6]. The parameter $a_{t} \equiv \xi a_{s}$ can be thought of as a "temporal lattice spacing," and additional irrelevant terms subleading in $a_{t}$ could be added, but the above symmetric form suits our purposes best. Eq. (1.1) implies that $\hat{U}$ acts as a raising operator for the electric quantum number, and can be expressed in the electric field basis as $\hat{U}=$ $\sum_{\varepsilon}|\varepsilon+1\rangle\langle\varepsilon|$. The action of $\hat{P}$, therefore, is to create an oriented loop of unit electric flux around the edge of the plaquette, while $\hat{P}^{\dagger}$ creates a unit loop in the opposite direction. At the same time, $\hat{P}$ measures magnetic field, the phase of its eigenvalue being the magnetic flux through the plaquette to leading order in $a_{s}$. The above form for $\hat{H}$ is bounded and written as a sum of unitary operators, which may be convenient for simulation by quantum walks [7].

Note that fluctuations in the magnetic field are large at strong coupling, while electric fluctuations are large at weak coupling. This is similar to the case of a harmonic oscillator with mass $m$ and spring constant $k$, where $\left\langle\hat{x}^{2}\right\rangle \propto 1 / \sqrt{k m}$, while $\left\langle\hat{p}^{2}\right\rangle \propto \sqrt{\mathrm{km}}$, the operators $\hat{x}, \hat{p}$ being analogues of $\hat{B}, \hat{E}$ respectively, while $m \sim 1 / \tilde{g}_{t}^{2}$ and $k \sim 1 / \tilde{g}_{s}^{2}$. This suggests that regulating the theory with a cutoff on electric field values is a poor choice for gauge theories in $d<3$, as the continuum limit occurs in the weak coupling limit.

The physical subspace $\mathcal{H}_{\text {phys }} \subset \mathcal{H}$ consists of those states obeying the Gauss law constraint $\vec{\nabla} \cdot \vec{E}=0$. On the lattice, the analogue constraint is that at each lattice site the product of the $\hat{Q}_{\text {s }}$ on each outgoing link and $\hat{Q}^{\dagger}$ s on each incoming link must equal the unit operator:

$$
\left.\left.\prod_{\ell \text { into } \mathbf{n}} \hat{Q}_{\ell} \prod_{\ell \text { out of } \mathbf{n}} \hat{Q}_{\ell}^{\dagger} \mid \text { phys }\right\rangle=\mid \text { phys }\right\rangle \text {. }
$$

Most states in $\mathcal{H}$ violate eq. (1.4) and are unphysical, and therefore simulating Hamiltonian evolution in $\mathcal{H}$ will use more qubits on a quantum computer than physically necessary. To better understand this constraint, consider the lattice $L$ with periodic boundary conditions in $d=2,3$ dimensions with $n$ sites, and therefore $\ell=n d$ links, $p=n d(d-1) / 2$ plaquettes, and $c=n d(d-1)(d-2) / 6$ cubes. The Hilbert space $\mathcal{H}$ is characterized by the eigenvalues of the $\ell$ electric field variables, $\hat{Q}_{\ell}$. States fall into topological sectors labeled by an integer-valued $d$-tuple, $\boldsymbol{v}=\left(v_{1}, \ldots, v_{d}\right)$ designating $v_{i}$ units of electric flux wrapping around the $\mathbf{e}_{i}$ direction of the lattice. For a given topological sector we have $(n+d-1)$ constraints on the $\ell=n d$ electric field variables: $(n-1)$ constraints from Gauss's law and $d$ from fixing the topology. Therefore there are $[n d-(n+d-1)]=(n-1)(d-1)$ variables to describe physical states in a particular topological sector. If we place a cutoff on electric field values to regulate the theory, and assume $n \gg 1$, then the minimum number of qubits required to describe $\mathcal{H}_{\text {phys }}$ will scale as $(d-1) / d$ times the minimum number required for $\mathcal{H}$; this ratio is expected to be significantly smaller for non-Abelian theories. 
The benefit of restricting a computation to $\mathcal{H}_{\text {phys }}$ is not only in reduction of qubits, but also in ensuring that computational errors do not propagate states into the unphysical part of $\mathcal{H}$, a process that would look like violation of charge conservation. A brute force approach for restricting $\mathcal{H} \rightarrow \mathcal{H}_{\text {phys }}$ is to eliminate the constrained variables by solving the operator constraint. (For more details on this solution, we refer the reader to [8].) A heavy price is paid, however, in the loss of locality and discrete translational invariance of the resulting Hamiltonian. We next describe an alternative procedure, which leads directly to a duality transformation.

\section{U(1) dual formulation}

Physical states can be defined in terms of gauge-invariant operators acting on the trivial state $|0\rangle$ with zero electric field everywhere [1]; those operators can be constructed out of Wilson loops - products of $\hat{U}_{\ell}$ along closed paths. We first define the Polyakov loop operators $\hat{W}\left(C_{i}\right)$ to be the product of oriented $\hat{U}$ link operators along a closed loop $C_{i}$ that wraps around the lattice in the $\mathbf{e}_{i}$ compact direction. We then define the state

$$
|\boldsymbol{v}\rangle \equiv \prod_{i=1}^{d}\left(\hat{W}\left(C_{i}\right)\right)^{v_{i}}|0\rangle, \quad v_{i} \in \mathbb{Z} .
$$

All the physical states within a topological class are then created by acting on $|\boldsymbol{v}\rangle$ with powers of plaquette operators:

$$
|\mathscr{A}\rangle_{\boldsymbol{v}}=\prod_{\boldsymbol{p}}\left(\hat{P}_{\boldsymbol{p}}\right)^{\mathscr{A}_{\boldsymbol{p}}}|\boldsymbol{v}\rangle, \mathscr{A}_{\boldsymbol{p}} \in \mathbb{Z}
$$

where $\boldsymbol{p}$ runs over all $p$ plaquette coordinates. It is evident that $|\mathscr{A}\rangle_{\boldsymbol{v}} \in \mathcal{H}_{\text {phys }}$ for all $\mathscr{A}$ and $\boldsymbol{v}$ since both the $\hat{W}$ and $\hat{P}$ operators are gauge-invariant, each producing only closed loops of electric flux. It is also easy to see that any of the $\otimes_{\ell}\left|\varepsilon_{\ell}\right\rangle$ basis states obeying Gauss's law can be written in this form. The particular choice of the $C_{i}$ paths is unimportant, since two such paths can be deformed into each other by the application of plaquette operators. The problem now, however, is that the $|\mathscr{A}\rangle_{v}$ states are an over-complete basis for $\mathcal{H}_{\text {phys }}$, since a state in a particular topological sector depends on $p=n d(d-1) / 2$ variables instead of the required $(n-1)(d-1)$. The number of redundant $\mathscr{A}$ variables is therefore $R=(d-1)[1+(d-2) n / 2]$. For $d=2$, the redundancy is $R=1$, independent of the number of sites $n$; for $d=3, R=2+n$, scaling with the volume of the lattice. These redundancies arise because the product of plaquette operators around any closed surface is an identity operation, expressing the discretized integral form of $\vec{\nabla} \cdot \vec{B}=0 ; R$ simply counts the number of independent closed surfaces. We will deal with the redundancy by treating all of the $|\mathscr{A}\rangle_{v}$ states as independent, then subsequently imposing the magnetic Gauss law constraint.

The action of the Hamiltonian eq. (1.2) on the $|\mathscr{A}\rangle_{v}$ states is simple to characterize: $\hat{H}_{B}$ applies plaquette operators to the state, and therefore either raises or lowers $\mathscr{A}_{p}$ by one. $\hat{H}_{E}$ measures the electric field, which at each link is determined by differences between the $\mathscr{A}_{\boldsymbol{p}}$ for plaquettes it borders - with a possible additional contribution from the Polyakov loop in eq. (2.1) if the link lies along one of the $C_{i}$ curves. $\hat{H}_{E}$ therefore looks like a finite difference operator acting on $\mathscr{A}_{\boldsymbol{p}}$. The behavior of $H_{B}$ and $H_{E}$ can be most naturally described in terms of operators on the dual lattice. 
We first discuss the simpler case of $d=2$, where the duality transformation maps the $\{\mathbf{n}, \boldsymbol{\ell}, \boldsymbol{p}\}$ coordinates of $L$ to $\left\{\boldsymbol{p}^{\star}, \ell^{\star}, \mathbf{n}^{\star}\right\}$, respectively, on the dual lattice $L^{\star}$. $\mathbf{n}$ sits at the center of plaquette $\boldsymbol{p}^{\star}$, and vice versa, while $\boldsymbol{\ell}^{\star}$ and $\boldsymbol{\ell}$ intersect each other; our convention is that $x$-links of the two lattices are oriented anti-parallel to each other, while the $y$-links are parallel. By ignoring the redundancy in our definition of $\left|\mathscr{A}_{\rangle}\right\rangle_{\boldsymbol{v}}$ in eq. (2.2), we can treat $\mathscr{A}_{\mathbf{n}^{\star}}$ as an independent variable on each site and use product states $\otimes_{\mathbf{n}^{\star}}\left|\mathscr{A}_{\mathbf{n}^{\star}}\right\rangle$ as a basis for a Hilbert space $\mathcal{H}^{\star}$. In terms of these states, we can define the two local coordinate and shift operators, $\hat{\mathscr{U}}_{\mathbf{n}^{\star}}$ and $\hat{\mathscr{Q}}_{\mathbf{n}^{\star}}$, living on sites of $L^{\star}$ as

$$
\hat{\mathscr{U}}_{\mathbf{n}^{\star}}=\sum_{\mathscr{A}_{\mathbf{n}^{\star}}}\left|\mathscr{A}_{\mathbf{n}^{\star}}\right\rangle e^{i \xi \mathscr{A}_{\mathbf{n}^{\star}}}\left\langle\mathscr{A}_{\mathbf{n}^{\star}}\left|, \quad \hat{\mathscr{Q}}_{\mathbf{n}^{\star}}=\sum_{\mathscr{A}_{\mathbf{n}^{\star}}}\right| \mathscr{A}_{\mathbf{n}^{\star}}+1\right\rangle\left\langle\mathscr{A}_{\mathbf{n}^{\star}}\right| .
$$

For a given topological sector $\boldsymbol{v}$, the matrix elements of the Hamiltonian $\hat{H}$ of eq. (1.2) between the $|\mathscr{A}\rangle_{\boldsymbol{v}}$ states are reproduced then by the dual Hamiltonian $\hat{\mathscr{H}}_{\boldsymbol{v}}$ on $L^{\star}$,

$$
\hat{\mathscr{H}}_{\boldsymbol{v}}=\frac{1}{2 a_{s}} \sum_{\mathbf{n}^{\star}}\left[\frac{1}{\tilde{g}_{s}^{2}}\left(2-\hat{\mathscr{Q}}_{\mathbf{n}^{\star}}-\hat{\mathscr{Q}}_{\mathbf{n}^{\star}}^{\dagger}\right)-\frac{\tilde{g}_{t}^{2}}{\xi^{2}} a_{s}^{2} \hat{\mathscr{U}}_{\mathbf{n}^{\star}}^{\dagger} \Delta \hat{\mathscr{U}}_{\mathbf{n}^{\star}}\right] \quad(d=2) .
$$

Above, $\Delta$ is a discrete covariant Laplacian $\Delta=D_{i}^{+} D_{i}^{-}$, where $D_{i}^{+}$are the difference operators

$$
\begin{aligned}
D_{1}^{+} F_{\mathbf{n}^{\star}} & =\left(\mathscr{W}_{\left\{\mathbf{n}^{\star}, \mathbf{n}^{\star}-\mathbf{e}_{1}\right\}} F_{\mathbf{n}^{\star}-\mathbf{e}_{1}}-F_{\mathbf{n}^{\star}}\right) / a_{s}, \\
D_{2}^{+} F_{\mathbf{n}^{\star}} & =\left(\mathscr{W}_{\left\{\mathbf{n}^{\star}, \mathbf{n}^{\star}+\mathbf{e}_{2}\right\}} F_{\mathbf{n}^{\star}+\mathbf{e}_{2}}-F_{\mathbf{n}^{\star}}\right) / a_{s},
\end{aligned}
$$

$D_{i}^{-} \equiv-\left(D_{i}^{+}\right)^{\dagger}$, and the discrete vector gauge field $\mathscr{W}$ accounts for the topological charges $v$ :

$$
\mathscr{W}_{\ell^{\star}}=e^{i \xi v_{i}}, \text { if } \ell \in C_{i} ; \quad 1, \text { otherwise; }
$$

$\ell^{\star}$ being the link dual to $\ell$. Note that $D_{1}^{+}$is a derivative in the $-\mathbf{e}_{1}$ direction because on $L^{\star}$ we have oriented the $x$-links anti-parallel to those on $L$, unlike the $y$-links, which are parallel. The gauge symmetry associated with $\mathscr{W}$ reflects the equivalence of constructions based on different $C_{i}$ paths for the Polyakov loops in eq. (2.1).

The first term in eq. (2.4) arises from $\hat{H}_{B}$, while the second from $\hat{H}_{E}$, and we see that the roles of the two have been reversed: $\hat{H}_{B}$ becomes an operator that translates the value of the dual field $\mathscr{A}$, while $\hat{H}_{E}$ measures gradients in $\mathscr{A}$. The discrete gauge field $\mathscr{W}$ corresponding to the topological electric fields of the original theory seems to have no analogue in the original theory, but that is simply because we did not build in topological magnetic field loops; to do so would require a field analogous to $\mathscr{W}$ added to the original Hamiltonian $\hat{H}$.

As mentioned above, there is one redundant variable arising from the fact that $\prod_{p} \hat{P}_{p}=\hat{\mathbf{1}}$. Thus the restriction to $\mathcal{H}_{\text {phys }} \subset \mathcal{H}^{\star}$ requires applying the single constraint on physical states

$$
\left(\hat{\mathscr{Q}}_{L^{\star}}-\hat{\mathbf{1}}\right)|\mathscr{A}\rangle_{\boldsymbol{v}}=0, \quad \hat{\mathscr{Q}}_{L^{\star}} \equiv \prod_{\mathbf{n}^{\star}} \hat{\mathscr{Q}}_{\mathbf{n}^{\star}}
$$

This constraint can be solved by setting $\mathscr{A}=0$ at a single site $\mathbf{n}^{\star}$ and equating $\hat{\mathscr{Q}}$ at that site to the product of $\hat{\mathscr{Q}}^{\dagger}$ over all the other sites - again at the cost of sacrificing locality and discrete translational invariance. A more attractive alternative is to work directly in $\mathcal{H}^{\star}$ and simply use an initial wave function that satisfies eq. (2.7). Unlike in the conventional formulation, where the 
number of constraints scales with the number of lattice sites, here with only a single unphysical variable, the problems of constructing the initial state obeying the constraint — or of subsequently wandering into the unphysical subspace due to computational error - should be vastly diminished compared to simulations in the original space $\mathcal{H}$ subject to eq. (1.4). Because there is one $\hat{\mathscr{Q}}_{\mathbf{n}^{\star}}, \hat{\mathscr{U}}_{\mathbf{n}^{\star}}$ variable pair per site on $L^{\star}$, as compared with two $\hat{Q}_{\ell}, \hat{U}_{\ell}$ variable pairs per site on $L$, we see the expected $(d-1) / d=1 / 2$ reduction in degrees of freedom, which should correspond to a similar reduction in the number of qubits required to characterize the system. Now, to better substantiate this reduction, we must regulate $\mathcal{H}^{\star}$ to make it finite-dimensional.

To regulate the dual theory in $d=2$ one cannot simply limit $\mathscr{A}_{\mathbf{n}^{\star}}$ to lie in the finite range $-N \leq$ $\hat{\mathscr{A}}_{\mathbf{n}^{\star}} \leq N$, taking $N \rightarrow \infty$ in the continuum limit: the operator $\hat{\mathscr{Q}}_{L^{\star}}$ shifts the $\hat{\mathscr{A}}_{\mathbf{n}^{\star}}$ field uniformly so that the constraint eq. (2.7) cannot hold in a space spanned by eigenstates of the $\hat{\mathscr{A}}_{\mathbf{n}^{\star}}$ with finite eigenvalues. Instead, one can regulate the eigenvalues of the unitary $\hat{\mathscr{Q}}_{\mathbf{n}^{\star}}$ operators, equivalent to placing a cutoff on magnetic field fluctuations in the original theory. The regulated Hamiltonian will then commute with the constraint, and an initial wave function chosen to satisfy the constraint eq. (2.7) will continue to do so as it evolves. Therefore, in $d=2$, there are several advantages to simulating $\hat{\mathscr{H}}_{\boldsymbol{v}}$ on a quantum computer instead of $\hat{H}$ : (i) the variables are scalars, rather than vectors, reducing the number of degrees of freedom by half; (ii) there is a single redundant variable, rather than the $(n-1)$ unphysical variables in the conventional formulation; (iii) it is natural to regulate magnetic fluctuations rather than electric, which is likely to converge more efficiently to the continuum limit.

The Hamiltonian in $d=3$ for the $|\mathscr{A}\rangle_{\boldsymbol{v}}$ states of eq. (2.2) can be constructed along similar lines [8]; one is again led to a duality transformation, interchanging the coordinates for sites, links, plaquettes and cubes from $L$ to $L^{\star}$ as $\{\mathbf{n}, \boldsymbol{\ell}, \boldsymbol{p}, \boldsymbol{c}\} \leftrightarrow\left\{\boldsymbol{c}^{\star}, \boldsymbol{p}^{\star}, \boldsymbol{\ell}^{\star}, \mathbf{n}^{\star}\right\}$. In particular, the plaquettes $\boldsymbol{p}$ on $L$ get mapped to the links $\ell^{\star}$ on $L^{\star}$ piercing them in the direction opposite to their normal vectors, so that $L^{\star}$ is parity inverted relative to $L$. Therefore the plaquette variable $\mathscr{A}_{\boldsymbol{p}}$ on $L$ gets mapped to a dual vector field $\mathscr{A}_{\ell^{\star}}$ living on the links of $L^{\star}$, unlike in $d=2$ where a scalar $\mathscr{A}_{\mathbf{n}^{\star}}$ lives on sites. We can then define link operators $\hat{\mathscr{U}}_{\ell^{\star}}$ and $\hat{\mathscr{Q}}_{\ell^{\star}}$ operators exactly as in eq. (2.3). The $|\mathscr{A}\rangle_{\boldsymbol{v}}$ states in eq. (2.2) are again over-complete, but the problem is more severe than in $d=2$ since the product of plaquette operators on the surface of any cube $c$ in $L$ should be an identity transformation, the number of cubes scaling with $n$. The constraint on the dual lattice to remove this degeneracy is (unsurprisingly) the dual of the electric Gauss law constraint eq. (1.4): the same equation with the substitution $\hat{Q}_{\ell} \rightarrow \hat{\mathscr{Q}}_{\ell^{\star}}$. Thus, we see a "conservation of difficulty" between the original and dual theories for $d=3$, each form of the theory having a Gauss law constraint of identical form. The one advantage of the dual formulation shared with $d=2$ is that regulating the eigenvalues of the $\hat{\mathscr{Q}}$ operators controls magnetic fluctuations, which we expect to be more efficient at weak coupling than a cutoff on the electric field.

\section{Conclusions}

We have focused here entirely on $U(1)$ gauge theories without matter and have shown the consequences of defining these theories on the space of gauge-invariant states. In particular, we found that this leads to a dual formulation subject to a magnetic Gauss law constraint. This result can lead to a substantial reduction of variables in $d=2$, but not in $d=3$; in both cases though it offers 
the opportunity to regulate the theory by limiting magnetic fluctuations rather than electric, which is expected to be advantageous in approaching the continuum limit in $d=2$, or studying the weak field limit in $d=3$. One can hope for a similar approach to regulating asymptotically-free gauge theories in $d=3$, for which the continuum limit is also at weak coupling. Extending the analysis to include charged matter fields and non-Abelian gauge symmetries is complicated by the fact that not all gauge-invariant states in the theory can be written in the form eq. (2.2); much previous work on related issues for non-Abelian gauge theories exists $[9,10,11,12]$ and could serve as a basis for quantum computations. Understanding such theories better, and developing the tools to efficiently represent these theories on a quantum computer and extrapolate to the continuum theory, remain as fascinating theoretical challenges to be tackled before one can contemplate solving outstanding computational problems in QCD.

\section{Acknowledgments}

We would like to thank N. Klco, J. Lombard, M. Savage, and L. Yaffe for useful conversations. This work was supported in part by DOE Grant No. DE-FG02-00ER41132 and by the Thomas L. and Margo G. Wyckoff Endowed Faculty Fellowship; JRS was also supported in part by the National Science Foundation Graduate Research Fellowship under Grant No. DGE-1256082 and by the Seattle Chapter of the Achievement Rewards for College Scientists Foundation.

\section{References}

[1] J. B. Kogut and L. Susskind, Hamiltonian Formulation of Wilson's Lattice Gauge Theories, Phys. Rev. D11 (1975) 395.

[2] T. Byrnes and Y. Yamamoto, Simulating lattice gauge theories on a quantum computer, Phys. Rev. A73 (2006) 022328 [quant-ph/ 0510027 ].

[3] E. Zohar and M. Burrello, Formulation of lattice gauge theories for quantum simulations, Phys. Rev. D91 (2015) 054506 [1409.3085].

[4] U.-J. Wiese, Towards Quantum Simulating QCD, Nucl. Phys. A931 (2014) 246 [1409. 7414$].$

[5] M. Creutz, Quarks, gluons and lattices, vol. 8. Cambridge University Press, 1983.

[6] D. Horn, M. Weinstein and S. Yankielowicz, Hamiltonian Approach to Z(N) Lattice Gauge Theories, Phys. Rev. D19 (1979) 3715.

[7] G. H. Low and I. L. Chuang, Hamiltonian simulation by qubitization, arXiv:1610.06546 .

[8] D. B. Kaplan and J. R. Stryker, Gauss's Law, Duality, and the Hamiltonian Formulation of U(1) Lattice Gauge Theory, 1806.08797.

[9] R. Anishetty and H. S. Sharatchandra, Duality transformation for non-Abelian lattice gauge theories, Phys. Rev. Lett. 65 (1990) 813.

[10] M. Mathur, The loop states in lattice gauge theories, Phys. Lett. B640 (2006) 292 [hep-lat/0510101].

[11] M. Mathur, Loop Approach to Lattice Gauge Theories, Nucl. Phys. B779 (2007) 32 [hep-lat/0702007].

[12] I. Raychowdhury, Low Energy Spectrum of SU(2) Lattice Gauge Theory: An Alternate Proposal via Loop Formulation, arXiv:1804.01304 . 